\title{
Review Article \\ Human Epidermal Growth Factor Receptor 2 (HER2) in Cancers: Overexpression and Therapeutic Implications
}

\author{
Nida Iqbal ${ }^{1}$ and Naveed Iqbal ${ }^{2}$ \\ ${ }^{1}$ Department of Medical Oncology, Dr. B. R. A. Institute Rotary Cancer Hospital, All India Institute of Medical Sciences, \\ New Delhi 110029, India \\ ${ }^{2}$ Department of Anaesthesia and Intensive Care Unit, Indraprastha Apollo Hospital, New Delhi 110076, India
}

Correspondence should be addressed to Nida Iqbal; nida.iqbal55@yahoo.com

Received 28 May 2014; Revised 18 August 2014; Accepted 19 August 2014; Published 7 September 2014

Academic Editor: Jamboor Vishwanatha

Copyright (c) 2014 N. Iqbal and N. Iqbal. This is an open access article distributed under the Creative Commons Attribution License, which permits unrestricted use, distribution, and reproduction in any medium, provided the original work is properly cited.

\begin{abstract}
Human epidermal growth factor receptor 2 (HER2) is a member of the epidermal growth factor receptor family having tyrosine kinase activity. Dimerization of the receptor results in the autophosphorylation of tyrosine residues within the cytoplasmic domain of the receptors and initiates a variety of signaling pathways leading to cell proliferation and tumorigenesis. Amplification or overexpression of HER 2 occurs in approximately $15-30 \%$ of breast cancers and $10-30 \%$ of gastric/gastroesophageal cancers and serves as a prognostic and predictive biomarker. HER2 overexpression has also been seen in other cancers like ovary, endometrium, bladder, lung, colon, and head and neck. The introduction of HER2 directed therapies has dramatically influenced the outcome of patients with HER2 positive breast and gastric/gastroesophageal cancers; however, the results have been proved disappointing in other HER2 overexpressing cancers. This review discusses the role of HER2 in various cancers and therapeutic modalities available targeting HER2.
\end{abstract}

\section{Introduction}

The human epidermal growth factor receptor (HER) family of receptors plays a central role in the pathogenesis of several human cancers. They regulate cell growth, survival, and differentiation via multiple signal transduction pathways and participate in cellular proliferation and differentiation. The family is made up of four main members: HER-1, HER-2, HER-3, and HER-4, also called ErbB1, ErbB2, ErbB3, and ErbB4, respectively [1]. All four HER receptors comprise a cysteine-rich extracellular ligand binding site, a transmembrane lipophilic segment, and an intracellular domain with tyrosine kinase catalytic activity [2]. Epidermal growth factor receptor (EGFR, ErbB1, and HER1) - the first receptor tyrosine kinase, was discovered by Carpenter and coworkers at Vanderbilt University, USA, in 1978 [3]. ErbB stands for its origin in the Erb-b gene responsible for avian erythroblastosis virus. The neu oncogene (also known as HER2, ErbB2, or p185) was discovered by a group of scientists at Massachusetts Institute of Technology, Rockefeller, and Harvard University $[4,5]$. The HER2 receptor is a 1255 amino acid, $185 \mathrm{kD}$ transmembrane glycoprotein located at the long arm of human chromosome 17 (17q12) [6]. HER2 is expressed in many tissues and its major role in these tissues is to facilitate excessive/uncontrolled cell growth and tumorigenesis [7-9].

\section{Function}

The HER receptors exist as monomers on the cell surface. Upon ligands binding to their extracellular domains, HER proteins undergo dimerization and transphosphorylation of their intracellular domains. HER2 has no known direct activating ligand and may be in an activated state constitutively or become active upon heterodimerization with other family members such as HER1 and HER3. Homoor heterodimerization results in the autophosphorylation of tyrosine residues within the cytoplasmic domain of the receptors and initiates a variety of signaling pathways, principally the mitogen-activated protein kinase (MAPK), phosphatidylinositol-4,5-bisphosphate 3-kinase (PI3K), and protein kinase $\mathrm{C}(\mathrm{PKC})$ resulting in cell proliferation, 


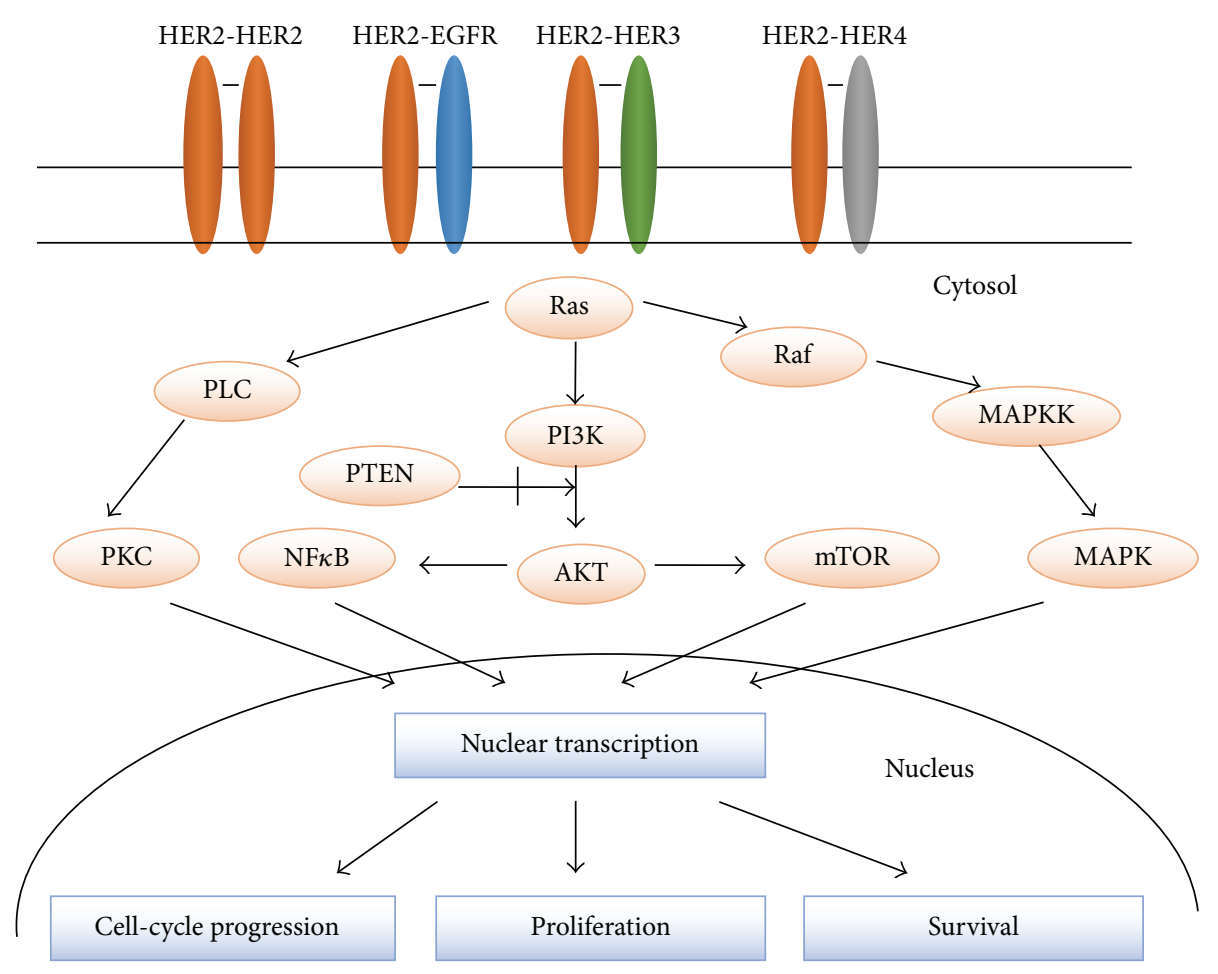

FIGURE 1: Receptor homodimerization or heterodimerization leads to activation of downstream signaling pathways promoting cell growth, proliferation, and survival. HER2 exists in an open conformation making it the dimerization partner of choice among the family members. The PI3K/AKT axis (which is regulated by PTEN and involves other key effectors such as NFאB and mTOR) and the Raf/MAPK cascade are the two most important and most extensively studied downstream signaling pathways that are activated by the HER receptors. Ras is at the top of these cascades and acts as a self-inactivating signal transducer. A third important factor in the network is PKC, which is activated by PLC. As a result of these signaling pathways, different nuclear factors are recruited and modulate the transcription of different genes involved in cell-cycle progression, proliferation, and survival. EGFR, epidermal growth factor receptor; HER, human epidermal growth factor receptor; PLC, phospholipase C; PKC, protein kinase C; PI3K, phosphatidylinositol 3-kinase; PTEN, phosphatase and tensin homolog; NF $\kappa$ B, nuclear factor $\kappa \mathrm{B}$; mTOR, mammalian target of rapamycin; MAPK, mitogen-activated protein kinase; MAPKK, MAPK kinase.

survival, differentiation, angiogenesis, and invasion. Heterodimers generate more potent signals than homodimers, and those containing HER2 have a particularly high ligand binding and signaling potency as HER2 exists in an open conformation making it the dimerization partner of choice among the family members. The HER2-HER3 heterodimer is the most potent stimulator of downstream pathways, particularly the PI3K/Akt, a master regulator of cell growth and survival. Moreover, HER2 dimerization promotes the mislocalization and rapid degradation of cell-cycle inhibitor p $27^{\text {Kipl }}$ protein leading to cell-cycle progression $[7,10,11]$. HER2 can also be activated by complexing with other membrane receptors such as insulin-like growth factor receptor 1 [12].

Figure 1 [13] shows the main transduction pathways regulated by the four HER family members-EGFR, HER2, HER3, and HER4.

\section{HER2 Overexpression in Cancers}

Most of the studies on HER2 have been carried out in breast cancer, after it was found to induce mammary carcinogenesis in vitro [14] and in vivo [15]. Amplification or overexpression of the HER2 gene occurs in approximately $15-30 \%$ of breast cancers [16]. With increasing understanding of HER2 biology, it has now been recognized that HER2 overexpression occurs in other forms of cancers also such as stomach, ovary, uterine serous endometrial carcinoma, colon, bladder, lung, uterine cervix, head and neck, and esophagus [17, 18]. Apart from its role in development of various cancers, it has also been intensely evaluated as a therapeutic target. The aim of this review is to update the role of HER2 in various cancers.

3.1. HER2 in Breast Cancer. HER2 is overexpressed in 15$30 \%$ of invasive breast cancers, which has both prognostic and predictive implications [16]. Breast cancers can have up to 2550 copies of the HER2 gene, and up to 40-100-fold increase in HER2 protein resulting in 2 million receptors expressed at the tumor cell surface [19]. Even estrogen, working via the nongenomic activity of estrogen receptor (ER) outside the nucleus, has been shown to activate HER2 signaling [20]. An aberrant form of HER2 (known as p95), lacking the extracellular domain, is found in some breast cancers. p95 is constitutively active and causes resistance to trastuzumab 
which requires the extracellular domain of HER2 for binding. For the same reason, p95 is not detected by antibodies that target the extracellular domain $[21,22]$.

HER2 gene amplification is associated with shorter disease-free and overall survival in breast cancer. Slamon et al. [23] established the prognostic significance of HER2 amplification in 189 human breast cancers. Amplification of HER2 gene was found to be a significant predictor of both overall survival $(P<0.001)$ and time to relapse $(P<0.0001)$. In a study by Press et al. [24], the expression of HER2 was studied in 704 node-negative breast cancers and it was found that women with breast cancer having high overexpression had a risk of recurrence 9.5 times greater than those whose breast cancers had normal expression $(P=0.0001)$. Analysis of various subgroups showed that the increased risk of recurrence extended across several subgroups of nodenegative breast cancer patients. Seshadri et al. [25] in their study of 1056 patients with Stages I-III breast cancer found that HER2 amplification 3-fold or greater was associated with significantly shorter disease-free survival $(P=0.0027)$. HER2 amplification also correlated significantly with pathologic stage of disease, number of axillary nodes with tumor, histologic type, and absence of estrogen receptor (ER) and progesterone receptor (PgR). Evidence suggests that HER2 amplification is an early event in human breast tumorigenesis. HER2 amplification is seen in nearly half of all in situ ductal carcinomas without any evidence of invasive disease and HER2 status is maintained during progression to invasive disease, nodal metastasis, and distant metastasis [26]. HER2 amplified breast cancers have increased sensitivity to certain cytotoxic chemotherapeutic agents and resistance to certain hormonal agents and increased propensity to metastasize to the brain [27].

3.2. HER2 in Gastric Cancer. HER2 overexpression in patients with gastric cancer has been reported from 10 to $30 \%$ and correlates with poor outcome and a more aggressive disease. Overexpression of HER2 protein in gastric cancer, using immunohistochemistry (IHC), was first described in 1986 [28]. In a study by Yano et al. [29], HER2 overexpression by IHC was found in $23 \%$ and gene amplification by FISH in $27 \%$ of 200 resected tumors. Gravalos and Jimeno [30] in their study of 166 gastric cancer patients observed that HER2 overexpression was most commonly found in gastroesophageal junction (GEJ) tumors and tumors having intestinal type histology. Other studies also confirmed a higher rate of HER2 positivity in GEJ tumors and intestinal subtype [31, 32]. HER2 overexpression is directly correlated with poorer outcome in gastric cancer. In a study of 260 gastric cancers, HER2 overexpression was an independent negative prognostic factor and HER2 staining intensity was correlated with tumor size, serosal invasion, and lymph node metastases [33]. Other studies also confirmed the negative impact of HER2 overexpression in gastric cancer $[34,35]$.

3.3. HER2 in Esophageal Cancer. HER2 overexpression is reported in $0-83 \%$ of esophageal cancers, with a tendency towards higher rates of positivity in adenocarcinoma (1083\%) compared to squamous cell carcinomas (0-56\%) [3639]. Yoon et al. [40], in their study of 713 patients with surgically resected esophageal adenocarcinomas (EAC), found HER2 positivity in $17 \%$ patients and it was significantly associated with lower tumor grade, less invasiveness, fewer malignant nodes, and the presence of adjacent Barrett's esophagus (BE). In EACs with Barrett's esophagus (BE), HER2 positivity was significantly associated with improved DSS $[\mathrm{HR}=0.54$ (95\% CI: 0.35-0.84), $P=0.0065]$ and overall survival $(P=0.0022)$ independent of pathologic features but was not prognostic among EACs without BE. However, another study by the same authors found that HER2 heterogeneity among HER2 amplified EACs was an independent predictor of worse cancer-specific survival [41]. Apart from EAC, HER2 overexpression was also found to be a negative predictor of survival in esophageal squamous cell carcinoma [42].

3.4. HER2 in Ovarian Cancer. Overexpression of HER2 is seen in $20-30 \%$ patients with ovarian cancer. Association of HER2 overexpression with poor survival in advanced epithelial ovarian cancer was first established by Berchuck et al. [43]. In a cohort of 73 patients with ovarian cancer, patients with HER2 overexpression had significantly worse survival as compared to patients with normal expression. In addition, patients whose tumors had high HER2 expression were significantly less likely to have a complete response to primary therapy or have a negative second-look laparotomy when serum CA 125 levels were normal preoperatively. Bartlett et al. [44] in their study of 76 patients with ovarian malignancy found that patients with tumors possessing EGF receptor mRNA had significantly reduced survival as compared to patients with tumors having negative expression. Although HER2 overexpression has been found to be associated with poorer survival, the usefulness of HER2 directed therapies is limited due to low frequency of strong expression.

3.5. HER2 in Endometrial Cancer. In endometrial serous carcinoma, the reported rates of HER2 overexpression range between $14 \%$ and $80 \%$ with HER2 amplification (by fluorescence in situ hybridization [FISH]) ranging from $21 \%$ to $47 \%$. HER2 overexpression and amplification in endometrioid carcinomas have been reported in the range from $1 \%$ to $47 \%$ and from $0 \%$ to $38 \%$, respectively [45-47]. Both HER2 overexpression and amplification have been linked to poor prognosis in endometrial carcinoma. Santin et al. [48] reported dramatically shorter overall survival in patients with HER2-amplified endometrial serous carcinoma compared with those without amplification. In addition, patients with high HER2 copy numbers (ratio >2.5) did significantly worse than those with lower HER2 amplification (ratio 2.0-2.5).

HER2 in Other Cancers. In lung cancers, overexpression of HER2 has been reported in about 20\% [49,50]. Apart from overexpression, mutations of HER2 were also reported in lung adenocarcinomas. The mutations targeted never or light smokers, oriental ethnicity, and female gender [51, 52]. 


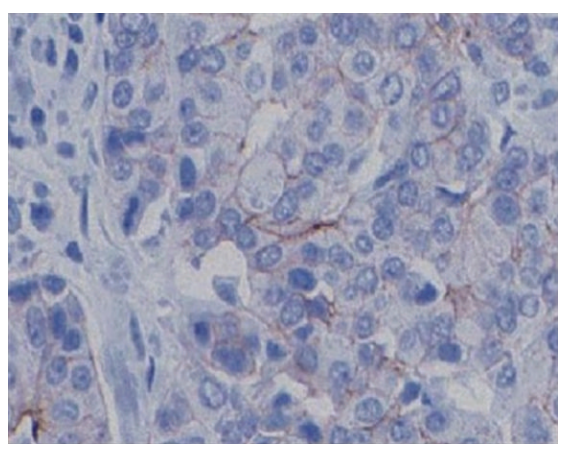

(a)

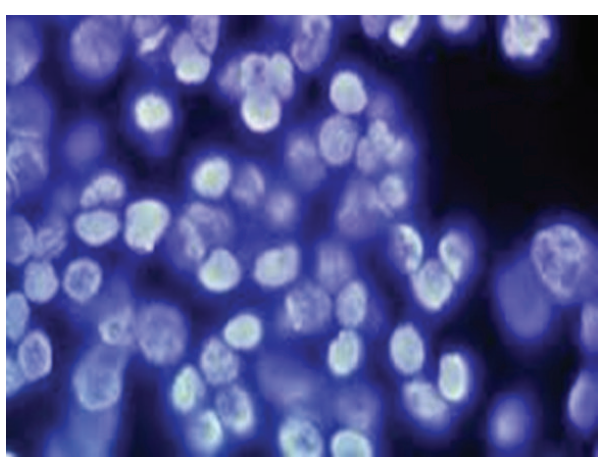

(c)

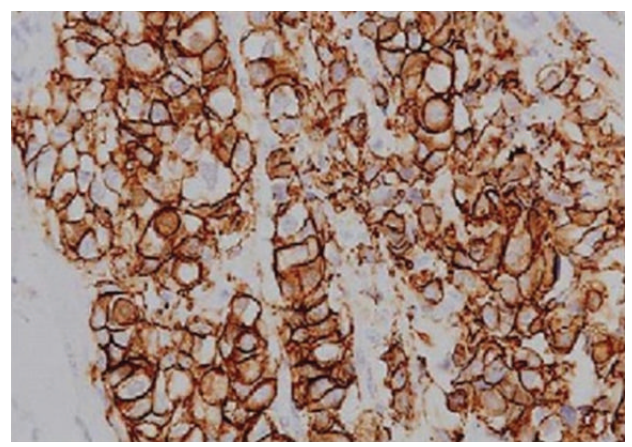

(b)

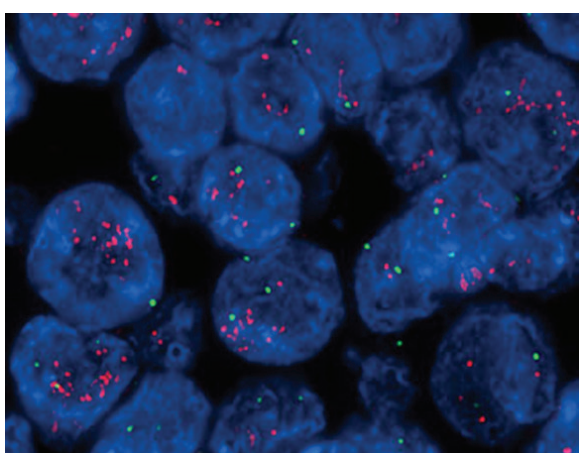

(d)

FIGURE 2: This image shows determination of HER2 status in 4 samples of breast tumor tissue. Samples (a) and (b) were analyzed by immunohistochemistry (IHC), while samples (c) and (d) were analyzed by fluorescence in situ hybridization (FISH). IHC detects protein and shows an increased expression of HER2 receptors, while FISH detects gene amplification. Sample (a) shows normal levels of HER2 protein expression, and sample (c) shows normal copy numbers of HER2 genes, while samples (b) and (d) show abnormal levels, respectively.

In invasive urothelial bladder carcinomas, amplification and/or overexpression range from $23 \%$ to $80 \%$ for overexpression and from $0 \%$ to $32 \%$ for amplification $[53,54]$. However, clinical trials using HER2 directed therapies in lung and bladder cancers reported disappointing clinical benefits $[55,56]$.

\section{Testing for HER2}

Although several methods for HER2 testing have been developed, approximately $20 \%$ of current HER2 testing may be inaccurate. Therefore, the American Society of Clinical Oncology (ASCO) and the College of American Pathologists (CAP) have recommended guidelines in HER2 testing to ensure accuracy [57]. The two methods currently approved for HER2 testing are immunohistochemistry (IHC) and fluorescence in situ hybridization (FISH).

Breast Cancer. HER2 status should be determined in all patients with invasive breast cancer on the basis of 1 or more test results. Breast cancer specimens should initially undergo HER2 testing by a validated immunohistochemistry (IHC) assay for HER2 protein expression [58]. The scoring method for HER2 expression is based on the cell membrane staining pattern and is as follows: (i) 3+: positive HER2 expression, uniform intense membrane staining of more than $30 \%$ of invasive tumor cells;

(ii) 2+: equivocal for HER2 protein expression, complete membrane staining that is either nonuniform or weak in intensity but has circumferential distribution in at least $10 \%$ of cells;

(iii) 0 or 1+: negative for HER2 protein expression.

Breast cancer specimens with equivocal IHC should undergo validation fluorescence in situ hybridization (FISH). The interpretation for HER2 FISH testing (HER2-to-CEP17 ratio and gene copy number) is as follows:

(i) positive HER2 amplification: FISH ratio higher than 2.2 or HER2 gene copy greater than 6.0;

(ii) equivocal HER2 amplification: FISH ratio of 1.8-2.2 or HER2 gene copy of 4.0-6.0;

(iii) negative HER2 amplification: FISH ratio lower than 1.8 or HER2 gene copy less than 4.0.

Figure 2 [59] shows HER2 analysis by IHC and FISH on breast tumor tissue.

Gastric Cancer. In gastric cancers, heterogeneity of the HER2 genotype can lead to discrepancies in the results 
TABLE 1: Consensus panel recommendations for HER2 scoring in gastric/esophageal cancer.

\begin{tabular}{llr}
\hline Score & Specimen & HER2 overexpression assessment \\
\hline 0 & $\begin{array}{l}\text { No reactivity or membranous reactivity in }<10 \% \text { of cells (resection): in biopsies only } \\
\text { one cohesive cluster of }>5 \text { cells is required. }\end{array}$ & Negative \\
$1+$ & $\begin{array}{l}\text { Faint membranous reactivity in }>10 \% \text { of tumor cells (resection): in biopsies only } \\
\text { one cohesive cluster of }>5 \text { cells is required. }\end{array}$ & Negative \\
$2+$ & $\begin{array}{l}\text { Weak to moderate incomplete (basolateral) membranous staining in }>10 \% \text { of tumor } \\
\text { cells (resection): in biopsies only one cohesive cluster of }>5 \text { cells is required. }\end{array}$ & Equivocal \\
$3+$ & $\begin{array}{l}\text { Moderate to strong incomplete (basolateral) membranous staining in }>10 \% \text { of } \\
\text { tumor cells (resection): in biopsies only one cohesive cluster of }>5 \text { cells is required. }\end{array}$ & Positive \\
\hline
\end{tabular}

from IHC and FISH testing [60]. Tumor heterogeneity was seen in roughly $4.8 \%$ of samples with moderate or strong HER2 staining and was higher than what was experienced in breast cancer (1.4\%) [61]. ASCO/CAP guidelines state that intratumoral heterogeneity may contribute to HER2 testing inaccuracy. Incomplete basolateral membrane HER2 IHC staining is also more common in gastric cancer than in breast cancer. This is due to the higher frequency of glandular formations that occur in gastric tissue. In gastric tissue, the basolateral membrane is stained, not the luminal membrane resulting in the heterogeneity. Currently, there are no ASCO/CAP approved HER2 testing guidelines for gastric cancer. Table 1 shows consensus panel recommendations on HER2 scoring for gastric/esophageal cancer [60, 61]. The National Comprehensive Cancer Network (NCCN) guidelines panel recommended that less than 3+ overexpression of HER2-neu by IHC should be additionally examined by FISH or other in situ hybridization methods. Gastric cancers with HER2 IHC overexpression of $3+$ or FISH positive are considered positive and thus be treated with trastuzumab. Thus, HER2 $3+$ or FISH +/HER2 IHC 1+, FISH +/HER2 IHC $2+$, FISH +/HER2 IHC 3+ gastric cancer patients should be treated with trastuzumab.

\section{Targeting HER2}

HER2 has been successfully targeted in breast cancer and gastric/gastroesophageal cancers. In ovarian cancer, HER2 is being investigated as a potential therapeutic target. There are several possible ways to target HER2.

5.1. Trastuzumab. Trastuzumab is a monoclonal antibody that binds to domain IV of the extracellular segment of the HER2 receptor. Proposed mechanisms of trastuzumab actions include (1) inhibition of HER2 shedding, (2) inhibition of PI3K-AKT pathway, (3) attenuation of cell signalling, (4) antibody-dependent cellular cytotoxicity, and (5) inhibition of tumor angiogenesis [62].

Trastuzumab was approved as part of a treatment regimen containing doxorubicin, cyclophosphamide, and paclitaxel for the adjuvant treatment of women with node-positive, HER2 overexpressing breast cancer. The approval was based on evidence of a significant prolongation in disease-free survival in women receiving trastuzumab and chemotherapy compared to those receiving chemotherapy alone. Table 2 shows five pivotal trials involving more than 10,000 women
TABLE 2: Five pivotal trials for adjuvant trastuzumab in breast cancer.

\begin{tabular}{|c|c|c|c|c|}
\hline Study & Control arm & $\begin{array}{c}\text { Trastuzumab } \\
\text { arm }\end{array}$ & $\begin{array}{l}\text { Reduction in } \\
\text { relative risk } \\
\text { of recurrence }\end{array}$ & $\begin{array}{c}\text { DFS } \\
\text { hazard } \\
\text { ratio }\end{array}$ \\
\hline $\begin{array}{l}\text { NSABP B-31 } \\
(N=2700)\end{array}$ & $\mathrm{AC} \rightarrow \mathrm{T}$ & $\mathrm{AC} \rightarrow \mathrm{TH}$ & \multirow{2}{*}{$\begin{array}{c}\text { Joint analysis } \\
52 \%\end{array}$} & \multirow{2}{*}{0.48} \\
\hline $\begin{array}{l}\text { NCCTG } \\
\text { N9831 } \\
(N=3300)\end{array}$ & $\mathrm{AC} \rightarrow \mathrm{T}$ & $\mathrm{AC} \rightarrow \mathrm{TH}$ & & \\
\hline $\begin{array}{l}\text { HERA } \\
(N=5090)\end{array}$ & Any & $\begin{array}{c}\text { Trastuzumab } \\
1 \text { year }\end{array}$ & $46 \%$ & 0.54 \\
\hline $\begin{array}{l}\text { BCIRG } 006 \\
(N=3150)\end{array}$ & $\mathrm{AC} \rightarrow \mathrm{D}$ & $\begin{array}{c}\mathrm{AC} \rightarrow \mathrm{DH} \\
\mathrm{DCH}\end{array}$ & $\begin{array}{l}40 \% \\
33 \%\end{array}$ & $\begin{array}{l}0.49 \\
0.61\end{array}$ \\
\hline $\begin{array}{l}\text { FINHer } \\
(N=232)\end{array}$ & $\begin{array}{l}\mathrm{D} \rightarrow \mathrm{FEC} \\
\mathrm{V} \rightarrow \mathrm{FEC}\end{array}$ & $\begin{array}{l}\mathrm{DH} \rightarrow \mathrm{FEC} \\
\mathrm{VH} \rightarrow \mathrm{FEC}\end{array}$ & & 0.42 \\
\hline
\end{tabular}

NSABP, National Surgical Adjuvant Breast and Bowel Project; NCCTG, North Central Cancer Treatment Group; HERA, Herceptin Adjuvant Trial; BCIRG, Breast Cancer International Research Group; FINHer, Finland Herceptin Study. A, doxorubicin; C, cyclophosphamide; D, docetaxel; E, epirubicin; F, fluorouracil; $\mathrm{H}$, trastuzumab; T, paclitaxel; V, vinorelbine; DFS, disease-free survival.

which demonstrated that one year of trastuzumab therapy provided significant clinical benefit [63-66]. These trials demonstrated that inclusion of trastuzumab produces roughly a 50\% improvement in disease-free survival and $33 \%$ improvement in overall survival, regardless of the chemotherapy regimen or sequence of trastuzumab delivery. In the metastatic HER2 breast cancer also, trastuzumab is recommended in the first-line setting. In a phase III trial, trastuzumab plus chemotherapy was associated with a significant improvement in time to disease progression, objective response rate, and 1-year survival compared with chemotherapy alone [67].

Trastuzumab was approved in combination with cisplatin and a fluoropyrimidine, for the treatment of patients with HER2 overexpressing metastatic gastric or gastroesophageal (GE) junction adenocarcinoma who have not received prior treatment for metastatic disease. The pivotal TOGA (trastuzumab for gastric cancer) trial demonstrated the median survival of 13.1 months for patients receiving trastuzumab and chemotherapy and 11.7 months for patients receiving chemotherapy alone. Trastuzumab was found to be most effective in prolonging survival in patients with HER2 
IHC 3+ tumors as compared to patients with IHC 2+ tumors [68].

Trastuzumab is recommended at a dose of $4 \mathrm{mg} / \mathrm{kg}$ followed by $2 \mathrm{mg} / \mathrm{kg}$ weekly for breast cancer and $8 \mathrm{mg} / \mathrm{kg}$ followed by $6 \mathrm{mg} / \mathrm{kg}$ q3 weekly for gastric/gastroesophageal cancer. The duration of therapy is one year in adjuvant setting for breast cancer and till disease progression for metastatic breast, gastric, and gastroesophageal cancer. The most common adverse effects seen with trastuzumab are fever, vomiting, infusion reactions, diarrhea, headache, fatigue, rash, neutropenia, and anemia. The most serious adverse effects include cardiomyopathy, pulmonary toxicity, infusion reactions, and febrile neutropenia. Left ventricular ejection fraction (LVEF) should be evaluated in all patients prior to and during treatment with trastuzumab.

5.2. Lapatinib. Lapatinib is an orally active dual tyrosine kinase inhibitor which interrupts the HER2 and epidermal growth factor receptor (EGFR) pathways. Lapatinib is approved in combination therapy with capecitabine for HER2 overexpressing advanced and metastatic breast cancer patients who have received prior therapy including an anthracycline, a taxane, and trastuzumab. This was based on a study that demonstrated delay in time to disease progression when lapatinib was used in combination with capecitabine. The risk of disease progression was reduced by $51 \%$, and the combination therapy was not associated with increases in toxic side effects [69]. Lapatinib is recommended at a dose of $1250 \mathrm{mg}$ PO qDay on days 1-21 continuously in combination with capecitabine $\left(2000 \mathrm{mg} / \mathrm{m}^{2} /\right.$ day PO divided $\left.\mathrm{q} 12 \mathrm{hr}\right)$ on days $1-14$ in a repeating 21 -day cycle.

Lapatinib is also approved in combination with leterozole for the treatment of postmenopausal women with hormone receptor and HER2 receptor positive metastatic breast cancers. The addition of lapatinib to letrozole is well tolerated and leads to a significantly greater progression free survival, overall response rate, and clinical benefit rate than with letrozole alone [70]. The most common adverse effects with lapatinib are diarrhea, anemia, hand-foot syndrome, liver dysfunction, nausea, rash, and neutropenia.

5.3. Pertuzumab. Pertuzumab is a humanized monoclonal antibody that blocks the activation of the HER2 receptor by hindering dimerization. Pertuzumab elicits action at a different ligand binding site from trastuzumab. It is approved in combination with trastuzumab and docetaxel in HER2positive metastatic breast cancer patients previously not treated with hormone therapy or chemotherapy. The approval of pertuzumab was based on results from the Clinical Evaluation of Pertuzumab and Trastuzumab (CLEOPATRA) trial. The trial compared first-line trastuzumab plus docetaxel (plus placebo) to trastuzumab plus docetaxel plus pertuzumab in HER2-positive metastatic breast cancer. Results from the study showed an average increase in progression-free survival of 6.1 months in patients receiving pertuzumab in addition to trastuzumab and docetaxel with minimal to no increase in cardiac toxic effects [71]. Pertuzumab is also approved for use as neoadjuvant treatment in combination with trastuzumab and docetaxel for patients with HER2positive, locally advanced, inflammatory, or early stage breast cancer. This was based on a randomized trial in which $39.3 \%$ of patients treated with pertuzumab, trastuzumab, and docetaxel achieved a pathologic complete response (pCR) compared with $21.5 \%$ of patients treated with trastuzumab and docetaxel at the time of surgery [72].

The recommended dose of pertuzumab is $840 \mathrm{mg}$ initial dose followed by $420 \mathrm{mg}$ every 3 weeks administered as an intravenous infusion over 30 to 60 minutes. The adverse effects seen with pertuzumab are alopecia, diarrhea, nausea, neutropenia, and cardiomyopathy.

5.4. Ado-Trastuzumab Emtansine. Ado-trastuzumab emtansine is an antibody-drug conjugate consisting of the monoclonal antibody trastuzumab linked to the cytotoxic agent mertansine (DM1). Most of the HER2-positive metastatic breast cancer patients eventually develop resistance. Adotrastuzumab offers a novel mechanism for overcoming trastuzumab resistance by exploiting trastuzumab to target the cytotoxic activity of DM1 to HER2 overexpressing cells. Ado-trastuzumab is approved as a single agent for treatment of HER2-positive, metastatic breast cancer in patients who have already received trastuzumab and a taxane either separately or in combination. Approval was based on results from EMILIA trial which compared ado-trastuzumab to lapatinib plus capecitabine. The study showed a significantly prolonged progression-free survival and overall survival with less toxicity than lapatinib plus capecitabine [73].

The recommended dose of ado-trastuzumab is $3.6 \mathrm{mg} / \mathrm{kg}$ IV infusion q3 weeks until disease progression or unacceptable toxicity. The most common adverse effects include fatigue, nausea, musculoskeletal pain, thrombocytopenia, headache, transaminitis, constipation, and peripheral neuropathy. The serious adverse effects include liver failure, hepatic encephalopathy, nodular regenerative hyperplasia, cardiac dysfunction, and interstitial lung disease.

5.5. Neratinib. Neratinib is an oral irreversible tyrosine kinase inhibitor of HER2 and EGFR. A phase II open label study in locally advanced breast cancer (LABC) showed a 16week progression-free survival rate of $75 \%$ in 36 trastuzumabnaive patients and $51 \%$ in previously treated disease [74]. Diarrhea was the most common grade $3 / 4$ toxic effect $(21 \%)$ in this study. Phase III evaluation of neratinib is ongoing in adjuvant trastuzumab-pretreated early-stage breast cancer.

5.6. Afatinib. Afatinib is an oral, irreversible inhibitor targeting EGFR/HER1, HER2, and HER4. Results from a phase II study of afatinib for HER2-positive MBC progressing posttrastuzumab $(N=41)$ showed 4 partial responses among 35 assessable patients. The most common all-grade treatmentrelated AEs included diarrhea (90.2\%) and rash (65.9\%) [75]. LUX-Breast 1 is an ongoing phase III study of vinorelbine plus either afatinib or trastuzumab for HER2-positive MBC in patients who failed one trastuzumab-containing regimen as first-line treatment of $\mathrm{MBC}$ or as adjuvant therapy [76]. 


\section{Conclusion}

HER2 has served as a prognostic and predictive biomarker in breast and gastric/gastroesophageal cancers. Therapies directed against HER2 have revolutionized the treatment of HER2 overexpressing breast and gastric cancers and improved the clinical outcome. Although HER2 overexpression was also found to correlate with poor outcome in other cancers, HER2 directed therapies provided disappointed results. Various novel HER2 directed agents alone or in combination are under investigation and in near future we will be expecting more varied implications of HER2 directed therapies. Till more robust data on the prognostic significance of HER2 in other cancers is available, HER2 testing and HER2 directed therapies are recommended in only breast and gastric/gastroesophageal cancers.

\section{Conflict of Interests}

The authors declare that there is no conflict of interests regarding the publication of this paper.

\section{References}

[1] D. J. Riese and D. F. Stern, "Specificity within the EGF family/ErbB receptor family signaling network," BioEssays, vol. 20, pp. 41-48, 1998.

[2] P. van der Geer, T. Hunter, and R. A. Lindberg, "Receptor protein-tyrosine kinases and their signal transduction pathways," Annual Review of Cell Biology, vol. 10, pp. 251-337, 1994.

[3] G. Carpenter, L. King Jr., and S. Cohen, "Epidermal growth factor stimulates phosphorylation in membrane preparations in vitro," Nature, vol. 276, no. 5686, pp. 409-410, 1978.

[4] L. C. Padhy, C. Shih, D. Cowing, R. Finkelstein, and R. A. Weinberg, "Identification of a phosphoprotein specifically induced by the transforming DNA of rat neuroblastomas," Cell, vol. 28 , no. 4 , pp. 865-871, 1982.

[5] A. L. Schechter, D. F. Stern, L. Vaidyanathan et al., "The neu oncogene: An erb-B-related gene encoding a 185,000- $\mathrm{M}_{r}$ tumour antigen," Nature, vol. 312, no. 5994, pp. 513-516, 1984.

[6] P. W. Brandt-Rauf, M. R. Pincus, and W. P. Carney, "The c-erbB2 protein in oncogenesis: molecular structure to molecular epidemiology," Critical Reviews in Oncogenesis, vol. 5, no. 2-3, pp. 313-329, 1994.

[7] M. A. Olayioye, "Update on HER-2 as a target for cancer therapy: intracellular signaling pathways of ErbB2/HER-2 and family members," Breast Cancer Research, vol. 3, no. 6, pp. 385389, 2001.

[8] R. M. Neve, H. A. Lane, and N. E. Hynes, "The role of overexpressed HER2 in transformation," Annals of Oncology, vol. 12, supplement 1, pp. S9-S13, 2001.

[9] S. Ménard, S. M. Pupa, M. Campiglio, and E. Tagliabue, "Biologic and therapeutic role of HER2 in cancer," Oncogene, vol. 22, no. 43, pp. 6570-6578, 2003.

[10] A. Citri and Y. Yarden, "EGF-ERBB signalling: towards the systems level," Nature Reviews Molecular Cell Biology, vol. 7, no. 7, pp. 505-516, 2006.

[11] M. M. Moasser, "The oncogene HER2: its signaling and transforming functions and its role in human cancer pathogenesis," Oncogene, vol. 26, no. 45, pp. 6469-6487, 2007.
[12] R. Nahta, L. X. H. Yuan, B. Zhang, R. Kobayashi, and F. J. Esteva, "Insulin-like growth factor-I receptor/human epidermal growth factor receptor 2 heterodimerization contributes to trastuzumab resistance of breast cancer cells," Cancer Research, vol. 65 , no. 23, pp. 11118-11128, 2005.

[13] L. Fornaro, M. Lucchesi, C. Caparello et al., "Anti-HER agents in gastric cancer: from bench to bedside," Nature Reviews Gastroenterology and Hepatology, vol. 8, no. 7, pp. 369-383, 2011.

[14] P. P. Di Fiore, J. H. Pierce, M. H. Kraus, O. Segatto, C. R. King, and S. A. Aaronson, "erbB-2 is a potent oncogene when overexpressed in NIH/3T3 cells," Science, vol. 237, no. 4811, pp. 178-182, 1987.

[15] W. J. Muller, E. Sinn, P. K. Pattengale, R. Wallace, and P. Leder, "Single-step induction of mammary adenocarcinoma in transgenic mice bearing the activated c-neu oncogene," Cell, vol. 54, no. 1, pp. 105-115, 1988.

[16] H. J. Burstein, "The distinctive nature of HER2-positive breast cancers," The New England Journal of Medicine, vol. 353, no. 16, pp. 1652-1654, 2005.

[17] S. I. Fukushige, K. I. Matsubara, M. Yoshida et al., "Localization of a novel v-erbB-related gene, c-erbB-2, on human chromosome 17 and its amplification in a gastric cancer cell line," Molecular and Cellular Biology, vol. 6, no. 3, pp. 955-958, 1986.

[18] U. Reichelt, P. Duesedau, M. Ch. Tsourlakis et al., "Frequent homogenous HER-2 amplification in primary and metastatic adenocarcinomas of the esophagus," Modern Pathology, vol. 20, no. 1, pp. 120-129, 2007.

[19] O.-P. Kallioniemi, A. Kallioniemi, W. Kurisu et al., "ERBB2 amplification in breast cancer analyzed by fluorescence in situ hybridization," Proceedings of the National Academy of Sciences of the United States of America, vol. 89, no. 12, pp. 5321-5325, 1992.

[20] J. Shou, S. Massarweh, C. K. Osborne et al., "Mechanisms of tamoxifen resistance: increased estrogen receptor-HER2/neu cross-talk in ER/HER2-positive breast cancer," Journal of the National Cancer Institute, vol. 96, no. 12, pp. 926-935, 2004.

[21] M. A. Molina, R. Sáez, E. E. Ramsey et al., "NH2-terminal truncated HER-2 protein but not full-length receptor is associated with nodal metastasis in human breast cancer," Clinical Cancer Research, vol. 8, no. 2, pp. 347-353, 2002.

[22] M. Scaltriti, F. Rojo, A. Ocaña et al., "Expression of p95HER2, a truncated form of the HER2 receptor, and response to antiHER2 therapies in breast cancer," Journal of the National Cancer Institute, vol. 99, no. 8, pp. 628-638, 2007.

[23] D. J. Slamon, G. M. Clark, S. G. Wong, W. J. Levin, A. Ullrich, and W. L. McGuire, "Human breast cancer: correlation of relapse and survival with amplification of the HER-2/neu oncogene," Science, vol. 235, no. 4785, pp. 177-182, 1987.

[24] M. F. Press, M. C. Pike, V. R. Chazin et al., "Her-2/neu expression in node-negative breast cancer: direct tissue quantitation by computerized image analysis and association of overexpression with increased risk of recurrent disease," Cancer Research, vol. 53, no. 20, pp. 4960-4970, 1993.

[25] R. Seshadri, F. A. Firgaira, D. J. Horsfall, K. McCaul, V. Setlur, and P. Kitchen, "Clinical significance of HER-2/neu oncogene amplification in primary breast cancer. The South Australian Breast Cancer Study Group," Journal of Clinical Oncology, vol. 11, no. 10, pp. 1936-1942, 1993.

[26] K. Park, S. Han, H. J. Kim, J. Kim, and E. Shin, "HER2 status in pure ductal carcinoma in situ and in the intraductal and invasive components of invasive ductal carcinoma determined by 
fluorescence in situ hybridization and immunohistochemistry," Histopathology, vol. 48, no. 6, pp. 702-707, 2006.

[27] Z. Gabos, R. Sinha, J. Hanson et al., "Prognostic significance of human epidermal growth factor receptor positivity for the development of brain metastasis after newly diagnosed breast cancer," Journal of Clinical Oncology, vol. 24, no. 36, pp. 56585663, 2006.

[28] K. Sakai, S. Mori, T. Kawamoto et al., "Expression of epidermal growth factor receptors on normal human gastric epithelia and gastric carcinomas," National Cancer Institute, vol. 77, no. 5, pp. 1047-1052, 1986.

[29] T. Yano, A. Ochiai, T. Doi et al., "Expression of HER2 in gastric cancer: comparison between protein expression and gene amplification using a new commercial kit," Journal of Clinical Oncology, vol. 22, article 14S, abstract 4053, 2004.

[30] C. Gravalos and A. Jimeno, "HER2 in gastric cancer: a new prognostic factor and a novel therapeutic target," Annals of Oncology, vol. 19, no. 9, pp. 1523-1529, 2008.

[31] Y.-J. Bang, E. van Cutsem, A. Feyereislova et al., "Trastuzumab in combination with chemotherapy versus chemotherapy alone for treatment of HER2-positive advanced gastric or gastrooesophageal junction cancer (ToGA): a phase 3, open-label, randomised controlled trial," The Lancet, vol. 376, no. 9742, pp. 687-697, 2010.

[32] M. Tanner, M. Hollmen, T. T. Junttila et al., "Amplification of HER-2 in gastric carcinoma: association with topoisomerase IIa gene amplification, intestinal type, poor prognosis and sensitivity to trastuzumab," Annals of Oncology, vol. 16, pp. 273278, 2005.

[33] Y. Yonemura, I. Ninomiya, A. Yamaguchi et al., "Evaluation of immunoreactivity for erbB-2 protein as a marker of poor shortterm prognosis in gastric cancer," Cancer Research, vol. 51, no. 3, pp. 1034-1038, 1991.

[34] S. Uchino, H. Tsuda, K. Maruyama et al., "Overexpression of cerbB-2 protein in gastric cancer. Its correlation with long-term survival of patients," Cancer, vol. 72, no. 11, pp. 3179-3184, 1993.

[35] M. Nakajima, H. Sawada, Y. Yamada et al., "The prognostic significance of amplification and overexpression of c-met and cerbB-2 in human gastric carcinomas," Cancer, vol. 85, pp. 18941902, 1999.

[36] J.-F. Flejou, F. Paraf, F. Muzeau et al., "Expression of c-erbB2 oncogene product in Barrett's adenocarcinoma: pathological and prognostic correlations," Journal of Clinical Pathology, vol. 47, no. 1, pp. 23-26, 1994.

[37] H. Safran, T. DiPetrillo, A. Nadeem et al., "Trastuzumab, paclitaxel, cisplatin, and radiation for adenocarcinoma of the esophagus: a phase I study," Cancer Investigation, vol. 22, no. 5, pp. 670-677, 2004.

[38] Z. Suo, W. Su, R. Holm, and J. M. Nesland, "Lack of expression of c-erbB-2 oncoprotein in human esophageal squamous cell carcinomas," Anticancer Research, vol. 15, no. 6B, pp. 2797-2798, 1995.

[39] M. Akamatsu, T. Matsumoto, K. Oka et al., "C-erbB-2 oncoprotein expression related to chemoradioresistance in esophageal squamous cell carcinoma," International Journal of Radiation Oncology Biology Physics, vol. 57, no. 5, pp. 1323-1327, 2003.

[40] H. H. Yoon, Q. Shi, W. R. Sukov et al., "Association of HER2/ErbB2 expression and gene amplification with pathologic features and prognosis in esophageal adenocarcinomas," Clinical Cancer Research, vol. 18, no. 2, pp. 546-554, 2012.

[41] H. H. Yoon, Q. Shi, W. R. Sukov et al., "Adverse prognostic impact of intratumor heterogeneous HER2 gene amplification in patients with esophageal adenocarcinoma," Journal of Clinical Oncology, vol. 30, no. 32, pp. 3932-3938, 2012.

[42] N. Zhan, W.-G. Dong, Y.-F. Tang, Z.-S. Wang, and C.-L. Xiong, "Analysis of HER2 gene amplification and protein expression in esophageal squamous cell carcinoma," Medical Oncology, vol. 29, no. 2, pp. 933-940, 2012.

[43] A. Berchuck, A. Kamel, R. Whitaker et al., "Overexpression of HER-2/neu is associated with poor survival in advanced epithelial ovarian cancer," Cancer Research, vol. 50, no. 13, pp. 4087-4091, 1990.

[44] J. M. S. Bartlett, S. P. Langdon, B. J. B. Simpson et al., "The prognostic value of epidermal growth factor receptor mRNA expression in primary ovarian cancer," British Journal of Cancer, vol. 73, no. 3, pp. 301-306, 1996.

[45] C. D. Rolitsky, K. S. Theil, V. R. McGaughy, L. J. Copeland, and T. H. Niemann, "HER-2/neu amplification and overexpression in endometrial carcinoma," International Journal of Gynecological Pathology, vol. 18, no. 2, pp. 138-143, 1999.

[46] C. Morrison, V. Zanagnolo, N. Ramirez et al., "HER-2 is an independent prognostic factor in endometrial cancer: association with outcome in a large cohort of surgically staged patients," Journal of Clinical Oncology, vol. 24, no. 15, pp. 2376-2385, 2006.

[47] T. A. Grushko, V. L. Filiaci, A. J. Mundt, K. Ridderstråle, O. I. Olopade, and G. F. Fleming, "An exploratory analysis of HER2 amplification and overexpression in advanced endometrial carcinoma: a gynecologic oncology group study," Gynecologic Oncology, vol. 108, no. 1, pp. 3-9, 2008.

[48] A. D. Santin, S. Bellone, S. van Stedum et al., "Amplification of cerbB2 oncogene: a major prognostic indicator in uterine serous papillary carcinoma," Cancer, vol. 104, no. 7, pp. 1391-1397, 2005.

[49] P. A. Bunn Jr., B. Helfrich, A. F. Soriano et al., "Expression of Her-2/neu in human lung cancer cell lines by immunohistochemistry and fluorescence in situ hybridization and its relationship to in vitro cytotoxicity by trastuzumab and chemotherapeutic agents," Clinical Cancer Research, vol. 7, no. 10, pp. 3239-3250, 2001.

[50] P. Heinmöller, C. Gross, K. Beyser et al., "HER2 status in non-small cell lung cancer: results from patient screening for enrollment to a phase II study of herceptin," Clinical Cancer Research, vol. 9, no. 14, pp. 5238-5244, 2003.

[51] P. Stephens, C. Hunter, G. Bignell et al., "Lung cancer: intragenic ERBB2 kinase mutations in tumours," Nature, vol. 431, pp. 525526, 2004.

[52] H. Shigemetsu, T. Takahashi, M. Nomura et al., "Somatic mutations of the HER2 kinase domain in lung adenocarcinomas," Cancer Research, vol. 65, article 1642, 2005.

[53] Z. Latif, A. D. Watters, I. Dunn, K. M. Grigor, M. Underwood, and J. Bartlett, "HER2/neu overexpression in the development of muscle-invasive transitional cell carcinoma of the bladder," British Journal of Cancer, vol. 89, no. 7, pp. 1305-1309, 2003.

[54] C. L. Coogan, C. R. Estrada, S. Kapur, and K. J. Bloom, "HER$2 /$ neu protein overexpression and gene amplification in human transitional cell carcinoma of the bladder," Urology, vol. 63, pp. 786-790, 2004.

[55] C. J. Langer, P. Stephenson, A. Thor, M. Vangel, and D. H. Johnson, "Trastuzumab in the treatment of advanced nonsmall-cell lung cancer: is there a role? Focus on Eastern Cooperative Oncology Group Study 2598," Journal of Clinical Oncology, vol. 22, no. 7, pp. 1180-1187, 2004.

[56] M. H. Hussain, G. R. MacVicar, D. P. Petrylak et al., "Trastuzumab, paclitaxel, carboplatin, and gemcitabine in 
advanced human epidermal growth factor receptor-2/neupositive urothelial carcinoma: results of a multicenter phase II National Cancer Institute trial," Journal of Clinical Oncology, vol. 25, no. 16, pp. 2218-2224, 2007.

[57] AC. Wolff, M. E. H. Hammond, D. G. Hicks et al., "Recommendations for human epidermal growth factor receptor 2 testing in breast cancer: American Society of Clinical Oncology/College of American Pathologists Clinical Practice Guideline Update," Journal Clinical Oncology, 2013.

[58] A. C. Wolff, M. E. Hammond, J. N. Schwartz et al., "American Society of Clinical Oncology/College of American Pathologists guideline recommendations for human epidermal growth factor receptor 2 testing in breast cancer," Journal of Clinical Oncology, vol. 25, no. 1, pp. 118-145, 2007.

[59] S. Ménard, E. Tagliabue, M. Campiglio, and S. M. Pupa, "Role of HER2 gene overexpression in breast carcinoma," Journal of Cellular Physiology, vol. 182, no. 2, pp. 150-162, 2000.

[60] M. Hofmann, O. Stoss, D. Shi et al., "Assessment of a HER2 scoring system for gastric cancer: results from a validation study," Histopathology, vol. 52, no. 7, pp. 797-805, 2008.

[61] J. Rüschoff, M. Dietel, G. Baretton et al., "HER2 diagnostics in gastric cancer-guideline validation and development of standardized immunohistochemical testing," Virchows Archiv, vol. 457, no. 3, pp. 299-307, 2010.

[62] T. Kute, C. M. Lack, M. Willingham et al., "Development of Herceptin Resistance in Breast Cancer Cells," Cytometry A, vol. 57, no. 2, pp. 86-93, 2004.

[63] E. H. Romond, E. A. Perez, J. Bryant et al., "Trastuzumab plus adjuvant chemotherapy for operable HER2-positive breast cancer," New England Journal of Medicine, vol. 353, no. 16, pp. 1673-1684, 2005.

[64] M. J. Piccart-Gebhart, M. Procter, B. Leyland-Jones et al., "Trastuzumab after adjuvant chemotherapy in HER2-positive breast cancer," The New England Journal of Medicine, vol. 353, no. 16, pp. 1659-1672, 2005.

[65] D. Slamon, W. Eiermann, N. Robert et al., "Phase III randomized trial comparing doxorubicin and cyclophosphamide followed by docetaxel $(\mathrm{AC} / \mathrm{T})$ with doxorubicin and cyclophosphamide followed by docetaxel and trastuzumab (AC/TH) with docetaxel, carboplatin and trastuzumab (TCH) in HER2 positive early breast cancer patients: BCIRG 006 study," Breast Cancer Research and Treatment, vol. 94, supplement 1, p. S1, 2005.

[66] H. Joensuu, P.-L. Kellokumpu-Lehtinen, P. Bono et al., "Adjuvant docetaxel or vinorelbine with or without trastuzumab for breast cancer," New England Journal of Medicine, vol. 354, no. 8, pp. 809-820, 2006.

[67] D. J. Slamon, B. Leyland-Jones, S. Shak et al., "Use of chemotherapy plus a monoclonal antibody against her2 for metastatic breast cancer that overexpresses HER2," The New England Journal of Medicine, vol. 344, no. 11, pp. 783-792, 2001.

[68] Y.-J. Bang, E. Van Cutsem, A. Feyereislova et al., "Trastuzumab in combination with chemotherapy versus chemotherapy alone for treatment of HER2-positive advanced gastric or gastrooesophageal junction cancer (ToGA): a phase 3, open-label, randomised controlled trial," The Lancet, vol. 376, no. 9742, pp. 687-697, 2010.

[69] C. E. Geyer, J. Forster, D. Lindquist et al., "Lapatinib plus capecitabine for HER2-positive advanced breast cancer," The New England Journal of Medicine, vol. 355, no. 26, pp. 2733$2743,2006$.
[70] L. S. Schwartzberg, S. X. Franco, A. Florance, L. O’Rourke, J. Maltzman, and S. Johnston, "Lapatinib plus letrozole as firstline therapy for HER-2+ hormone receptor-positive metastatic breast cancer," Oncologist, vol. 15, no. 2, pp. 122-129, 2010.

[71] J. Baselga, J. Cortés, S.-B. Kim et al., "Pertuzumab plus trastuzumab plus docetaxel for metastatic breast cancer," The New England Journal of Medicine, vol. 366, no. 2, pp. 109-119, 2012.

[72] L. Gianni, T. Pienkowski, Y.-H. Im et al., "Efficacy and safety of neoadjuvant pertuzumab and trastuzumab in women with locally advanced, inflammatory, or early HER2-positive breast cancer (NeoSphere): a randomised multicentre, open-label, phase 2 trial," The Lancet Oncology, vol. 13, no. 1, pp. 25-32, 2012.

[73] S. Verma, D. Miles, L. Gianni et al., "Trastuzumab emtansine for HER2-positive advanced breast cancer," The New England Journal of Medicine, vol. 367, no. 19, pp. 1783-1791, 2012.

[74] H. J. Burstein, Y. Sun, L. Y. Dirix et al., "Neratinib, an irreversible ErbB receptor tyrosine kinase inhibitor, in patients with advanced ErbB2-positive breast cancer," Journal of Clinical Oncology, vol. 28, no. 8, pp. 1301-1307, 2010.

[75] N. U. Lin, E. P. Winer, D. Wheatley et al., "A phase II study of afatinib (BIBW 2992), an irreversible ErbB family blocker, in patients with HER2-positive metastatic breast cancer progressing after trastuzumab," Breast Cancer Research and Treatment, vol. 133, no. 3, pp. 1057-1065, 2012.

[76] N. Harbeck, S.-A. Im, C. S. Huang et al., "LUX-breast 1: randomized, phase III trial of afatinib and vinorelbine versus trastuzumab and vinorelbine in patients with HER2overexpressing metastatic breast cancer (MBC) failing one prior trastuzumab treatment," Journal of Clinical Oncology, vol. 30, abstract TPS649, 2012. 

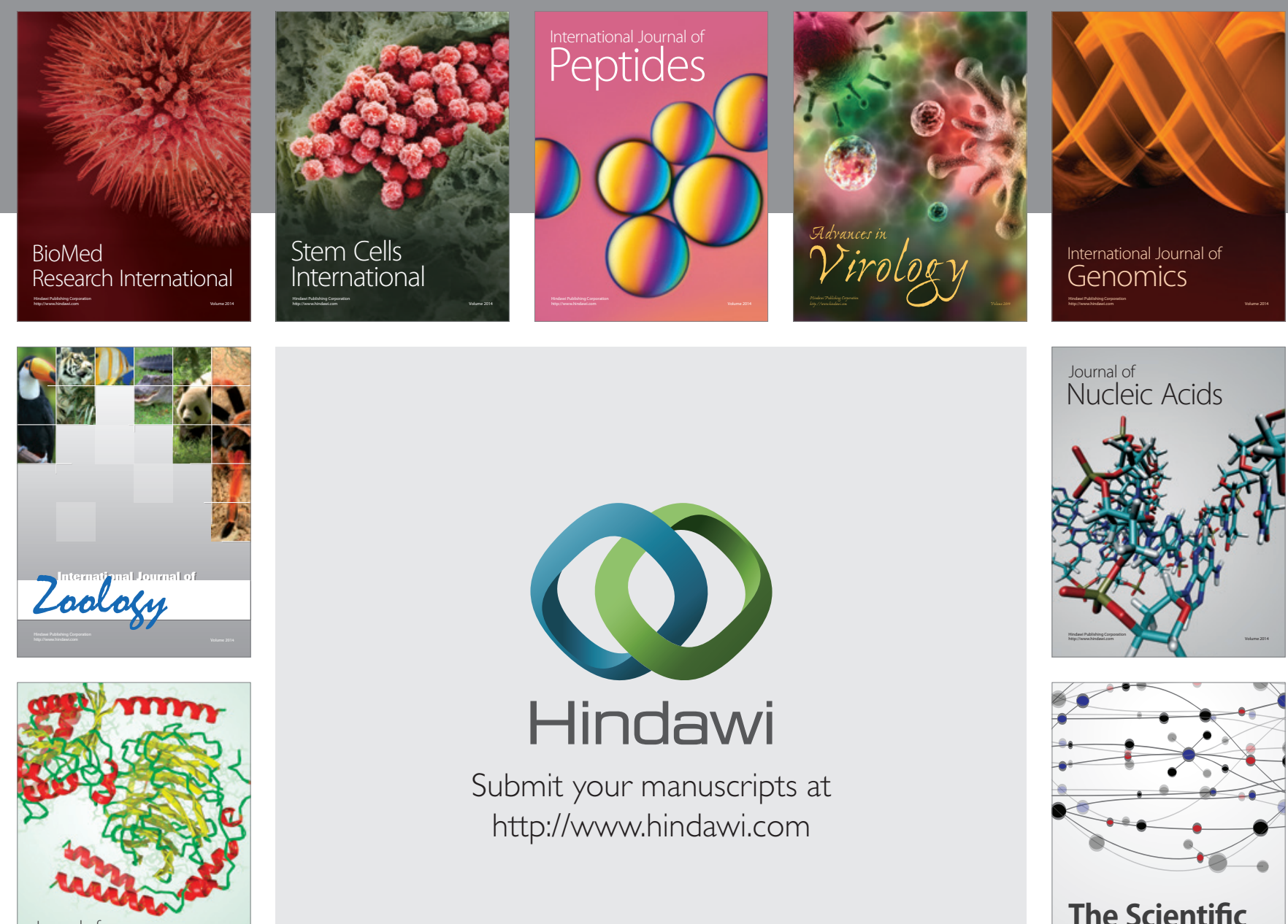

Submit your manuscripts at

http://www.hindawi.com

Journal of
Signal Transduction
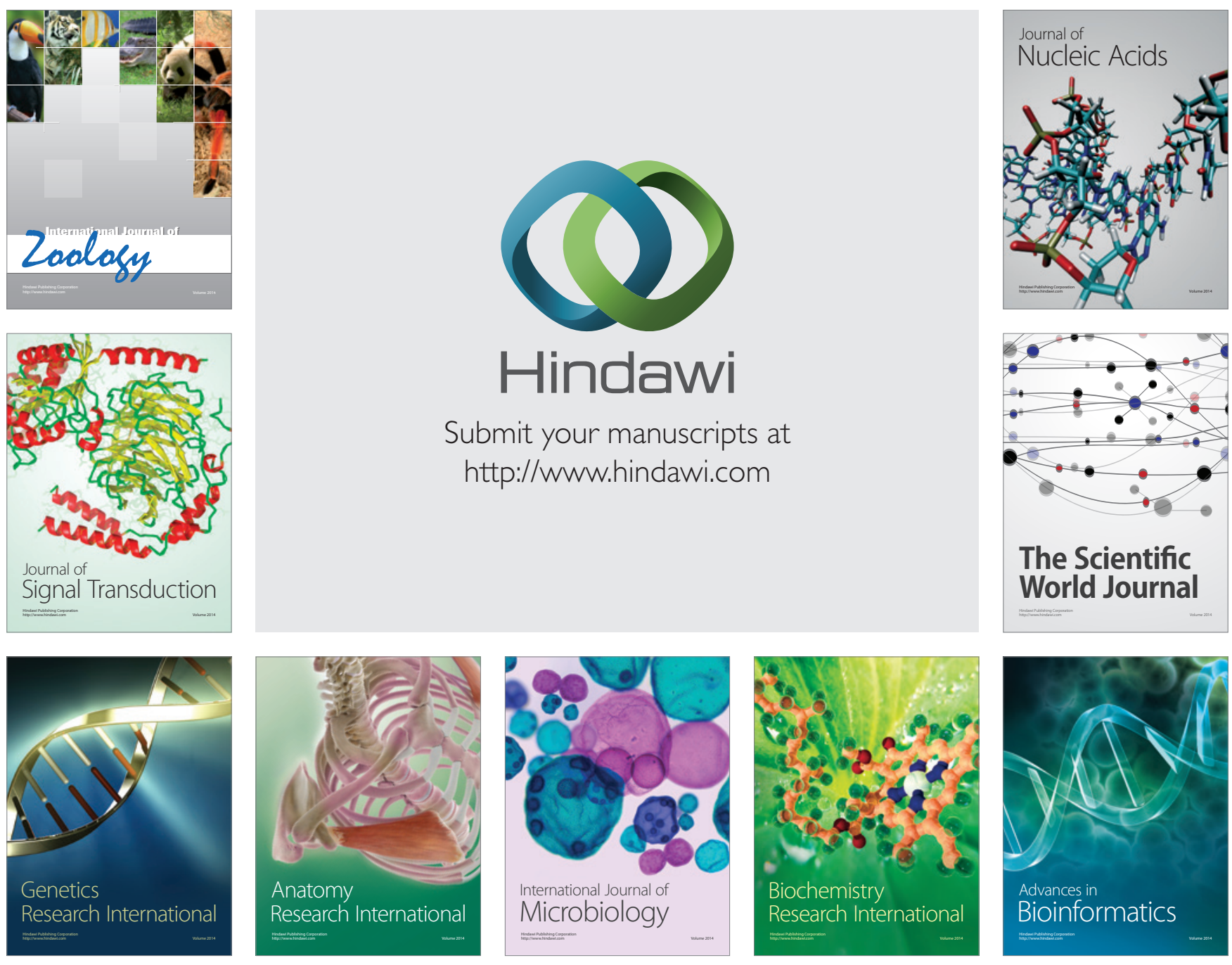

The Scientific World Journal
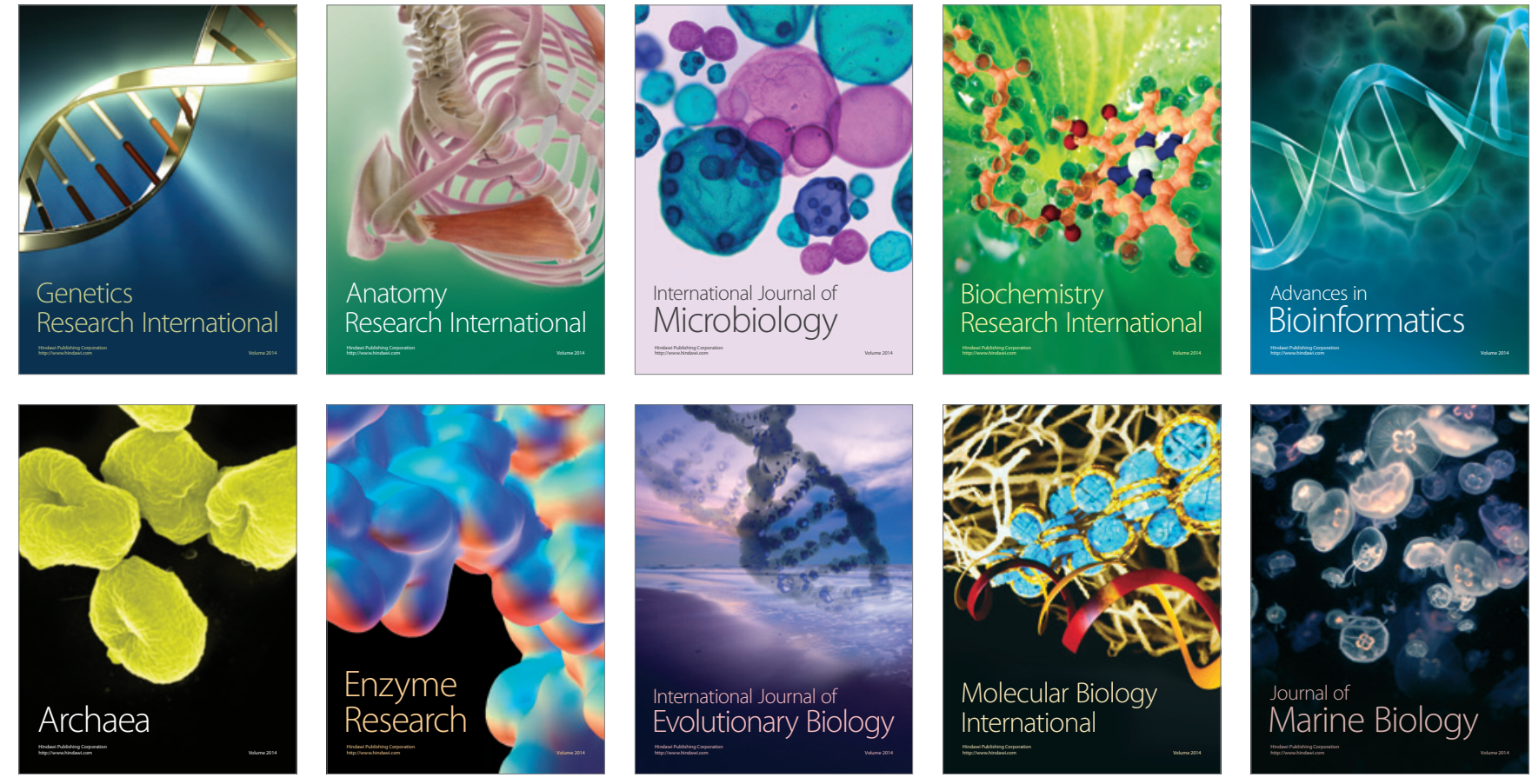